\title{
High Fundamental Repetition Rate Fiber Lasers Operated in Strong Normal Dispersion Regime
}

\author{
L. M. Zhao, C. Lu, Member, IEEE, H. Y. Tam, Senior Member, IEEE, P. K. A. Wai, Senior Member, IEEE, and \\ D. Y. Tang
}

\begin{abstract}
We demonstrate a high fundamental repetition rate erbium-doped fiber ring laser operated in strong normal dispersion regime passively mode-locked by the nonlinear polarization rotation technique. A novel scheme of using a polarization beam splitter as both the pump input coupler and the fiber laser output coupler is employed to increase the repetition rate of the fiber laser. Gain-guided solitons with a fundamental repetition rate of $234 \mathrm{MHz}$ and 1.11-ps pulsewidth are achieved from the ring laser cavity.
\end{abstract}

Index Terms-Optical fiber lasers, ultrafast optics.

\section{INTRODUCTION}

H IGH repetition rate fiber lasers are attractive for numerous applications, such as optical frequency metrology [1], high-speed optical sampling [2], and laser ranging [3]. Actively mode-locked lasers and harmonically passively mode-locked lasers can generate pulse trains with repetition rates well over $1 \mathrm{GHz}$ [4], [5]. However, actively mode-locked lasers need a radio-frequency $(\mathrm{RF})$ signal generator and a phase/intensity modulator, which results in a complicated structure and higher cost. The harmonically passively mode-locked lasers are relatively unstable and the timing jitter is larger than that of fundamentally passive mode locking. Therefore, passively mode-locked lasers running at high fundamental repetition rates are desirable.

Passively mode-locked lasers based on $\mathrm{Er}: \mathrm{Yb}$ : glass as a gain medium have culminated in fundamental repetition rates as high as $77 \mathrm{GHz}$ [6]. However, a high fundamental repetition rate fiber laser, in contrast to a glass- or crystal-based laser, would be advantageous due to its compact size, robustness, flexibility, low cost, and compatibility with other optical fiber systems.

Manuscript received December 23, 2008; revised March 02, 2009. First published March 24, 2009; current version published May 15, 2009. This work was supported by the Central Research Grant of the Hong Kong Polytechnic University under project G-YXOR.

L. M. Zhao was with the Department of Electronic and Information Engineering, The Hong Kong Polytechnic University, Hong Kong, China. He is now with the School of Electrical and Electronic Engineering, The Nanyang Technological University, 639798 Singapore, Singapore (e-mail: luming_zhao@pmail. ntu.edu.sg).

C. Lu and P. K. A. Wai are with the Department of Electronic and Information Engineering, The Hong Kong Polytechnic University, Hong Kong, China (e-mail: enluchao@polyu.edu.hk; enwai@ polyu.edu.hk).

H. Y. Tam is with the Department of Electrical Engineering, The Hong Kong Polytechnic University, Hong Kong, China (e-mail: eehytam@polyu.edu.hk).

D. Y. Tang is with the School of Electrical and Electronic Engineering, The Nanyang Technological University, 639798 Singapore, Singapore (e-mail: edytang@ntu.edu.sg).

Digital Object Identifier 10.1109/LPT.2009.2017277
The usual technique used for passive mode locking of fiber lasers relies on intensity-dependent phase shifts accumulated by the pulse as it propagates through the fiber. The ultrafast amplitude modulation converting from the nonlinear phase modulation can be achieved through an interferometer, as in the figure of eight fiber laser [7], or through an intracavity polarizer [8]. The fiber lasers using nonlinear fiber loop mirrors [7] and its variants are excluded from high fundamental repetition rate operation as they require longer fiber length. In fact, fiber lasers with linear cavity configurations employing a semiconductor saturable absorber mirror have achieved a fundamental repetition frequency of $2.08 \mathrm{GHz}$; however, the pulse dynamics is determined by the saturable absorber mirror and the spectral bandwidth of the pulse is limited to below $2.6 \mathrm{~nm}$ [9].

The high fundamental repetition rate fiber laser based on the nonlinear polarization rotation (NPR) combined with an intracavity polarizing element have attracted continuously strong research interest and fundamental repetition rates up to $200 \mathrm{MHz}$ in Yb-doped fiber laser [10] and $250 \mathrm{MHz}$ in Er-fiber laser [11] have been realized. A 194-MHz soliton Er-fiber laser with low timing jitter of $18 \mathrm{fs}(1 \mathrm{kHz}, 10 \mathrm{MHz})$ and low intensity noise was also reported [12]. However, these fiber lasers are operated in the stretched-pulse regime with anomalous net cavity dispersion [10], [11] or directly in the anomalous dispersion regime [12], where the pulse shaping mechanism is the balanced interaction between the cavity dispersion effect and the fiber nonlinear Kerr effect.

Recent research has revealed that the mode locking of fiber lasers can also be achieved in the normal dispersion regime, where the gain bandwidth limitation plays a crucial role for the mode locking [13]. The cumulative phase contribution of the cavity dispersion and the nonlinear effect is counteracted by the gain dispersion effect; consequently the gain-guided solitons are generated. In Yb-fiber lasers, with the help of an intracavity narrow bandpass filter, mode locking can also be achieved in the normal cavity dispersion regime [14]. The newly discovered mode locking mechanism in the normal dispersion regime seems to be promising for the progress of high fundamental repetition rate fiber lasers as most of the high gain fibers have largely normal dispersion [9]-[11], [13], [14] and the nonrequirement for the components providing anomalous dispersion greatly reduces the cavity length. However, as far as we know, no high fundamental repetition rate fiber laser operated in the largely normal dispersion regime has been reported.

In this letter, we revisit the limitation for high fundamental repetition rate operation of fiber lasers exploiting the NPR technique. Both physical size and mode-locking mechanism are re- 


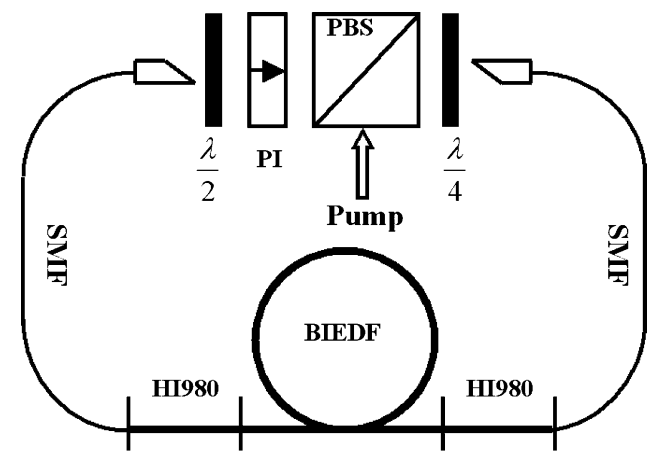

Fig. 1. Schematic of the fiber laser. $\lambda / 2$ : half-wave plate; PI: polarizationdependent isolator; $\lambda / 4$ : quarter-wave plate; BIEDF: $\mathrm{Bi}_{2} \mathrm{O}_{3}$-based erbium-doped fiber.

vised. We demonstrate an erbium-doped fiber laser with over 230-MHz fundamental repetition rate based on the gain-guided soliton operation. Potential for scaling up of the fundamental repetition rate of the fiber lasers is also discussed.

\section{LASER SCHEMATIC AND EXPERIMENTAL RESULTS}

The experimental setup is shown in Fig. 1. To match-up the available laser diode with maximum output power of $480 \mathrm{~mW}$, a 33.5-cm-long $\mathrm{Bi}_{2} \mathrm{O}_{3}$-based erbium-doped fiber (EDF) provided by Asahi Glass with group velocity dispersion (GVD) of about $-124(\mathrm{ps} / \mathrm{nm}) / \mathrm{km}$ at $1560 \mathrm{~nm}$ is used as the gain medium in the cavity. The erbium-ion concentration of the $\mathrm{Bi}_{2} \mathrm{O}_{3}$-based EDF is about $6470 \mathrm{ppm}$, which gives a $167-\mathrm{dB} / \mathrm{m}$ peak absorption around $1480 \mathrm{~nm}$. The typical nonlinear coefficient of the $\mathrm{Bi}_{2} \mathrm{O}_{3}$-based $\mathrm{EDF}$ is $50 \mathrm{~W}^{-1} \cdot \mathrm{km}^{-1}$. The core refractive index of the $\mathrm{Bi}_{2} \mathrm{O}_{3}$-based EDF is 2.03. To minimize the cavity length, different from previous setups [10], [12], the fiber-type wavelength-division-multiplexing coupler is removed from the fiber laser. Instead, we use a polarization beam splitter (PBS) to introduce the linearly polarized 1480 -nm pump laser (Fitel FOL1425RUZ) into the cavity while the PBS serves also as the fiber laser output coupler. Experimental measurement shows that the pump coupling loss through the PBS is about $0.8 \mathrm{~dB}$. The NPR technique is used to achieve the self-started mode locking in the laser. A polarization-dependent isolator is inserted in the cavity to allow unidirectional operation of the laser. Half-wave and quarter-wave plates are used to adjust the polarization of the light. The PBS, polarization-dependent isolator, and wave plates are mounted on an $8-\mathrm{cm}-$ long fiber bench. The lengths of the two standard single-mode fiber (SMF) pigtails attached to the fiber bench are 11.5 and $12.0 \mathrm{~cm}$, respectively. There are two segments of Corning HI980 fiber with GVD of about $-2.16(\mathrm{ps} / \mathrm{nm}) / \mathrm{km}$ function as a bridge fiber between the $\mathrm{Bi}_{2} \mathrm{O}_{3}$-based EDF and the SMF with 4.0-cm length each. The total cavity dispersion is estimated to be about $0.048 \mathrm{ps}^{2}$ at $1560 \mathrm{~nm}$.

Self-started mode locking can be achieved with a pump power of about $450 \mathrm{~mW}$ provided that the orientations of the wave plates are appropriately set. Single pulse mode-locking operation can be maintained with the pump power as low as $420 \mathrm{~mW}$. A series of measurements pertaining to the laser with 450-mW pump power are shown in Fig. 2. The pulse train with uniform amplitude is shown in Fig. 2(a). As we used a 1-GHz photodetector (New Focus 1611) and a 500-MHz oscilloscope (Tektronix TDS 714L) to measure the pulse train, the minor variation of pulse height can be attributed to the insufficient resolution of the oscilloscope. The period of the pulse train is about $4.27 \mathrm{~ns}$, that is, $234.2 \mathrm{MHz}$, which agrees well with that expected from the cavity length. Combined examination of the individual pulses, both with a 500-GHz sampling oscilloscope (Ando AQ7750) and an autocorrelator (FEMTOCHROME FR103-MN), shows no sign of intrapulse structure. The RF spectrum of Fig. 2(b) shows the simultaneous measurement corresponding to Fig. 2(a), more than 60-dB signal-to-noise ratio is obtained. The characteristically steep spectral edges as shown in Fig. 2(c) clearly suggest that the generated pulses are gain-guided solitons [13]. The linear spectrum suggests an asymmetric optical spectrum. Fig. 2(d) shows the autocorrelation traces of the original output pulse and the optimally compressed one. The 3-dB bandwidth is $14.7 \mathrm{~nm}$ and the pulsewidth is $1.11 \mathrm{ps}$ if a Gaussian pulse profile is assumed. We directly spliced a segment of SMF to compress the pulsewidth with cutting back the SMF length. The narrowest pulsewidth obtained is about $286 \mathrm{fs}$ with an optimized SMF length of $\sim 12 \mathrm{~m}$. No obvious difference is observed for the optical spectrum between the original output pulse and the compressed one. Therefore, the minimum time-bandwidth-product is about 0.526 , slightly larger than the transform-limited value of 0.44 . The pedestal shown in Fig. 2(d) is the consequence of the nonlinear chirp accumulated when the pulse propagates in the laser. The output power is about $25.1 \mathrm{~mW}$ with the pump power of $450 \mathrm{~mW}$, which corresponds a $0.107-n J$ pulse energy.

\section{DISCUSSION}

There are two main challenges for increasing the repetition rate of fiber lasers. First, the maximum repetition rate is ultimately dictated by the physical size of the components constituting the laser cavity. Second, a suitable mode-locking mechanism with moderate pump power requirements is crucial for the fiber laser. Our fiber laser design well overcomes these two obstacles. The PBS in the laser cavity functions as both the pump power input coupler and the fiber laser output coupler, therefore, the cavity length is comprised of only two parts: the free-space part and the gain fiber part. If the gain fiber can be directly attached to both ends of the fiber bench, then the fiber laser will become a gain-fiber-only fiber laser, which has the physically achievable minimum cavity length. We expect that the repetition rate can be increased to $600 \mathrm{MHz}$ with a $10-\mathrm{cm}$ free space region and 20-cm $\mathrm{Bi}_{2} \mathrm{O}_{3}$-based EDF. Ilday et al. [10] have studied the influence of the net cavity dispersion on the fiber laser mode locking and found the failure of mode locking at zero and positive dispersion regime. As the fiber laser were mode locked in the negative net cavity dispersion region, the free space part to compensate the fiber positive dispersion at $1 \mu \mathrm{m}$ cannot be omitted, which hinders the progress of increasing the fiber laser repetition rate. As in our case there is no need to add the components with negative dispersion to shift the net cavity dispersion into the negative dispersion regime for conventional soliton mode locking, cavity length could be greatly reduced especially for the proposed gain-fiber-only fiber laser. Our experiment has 

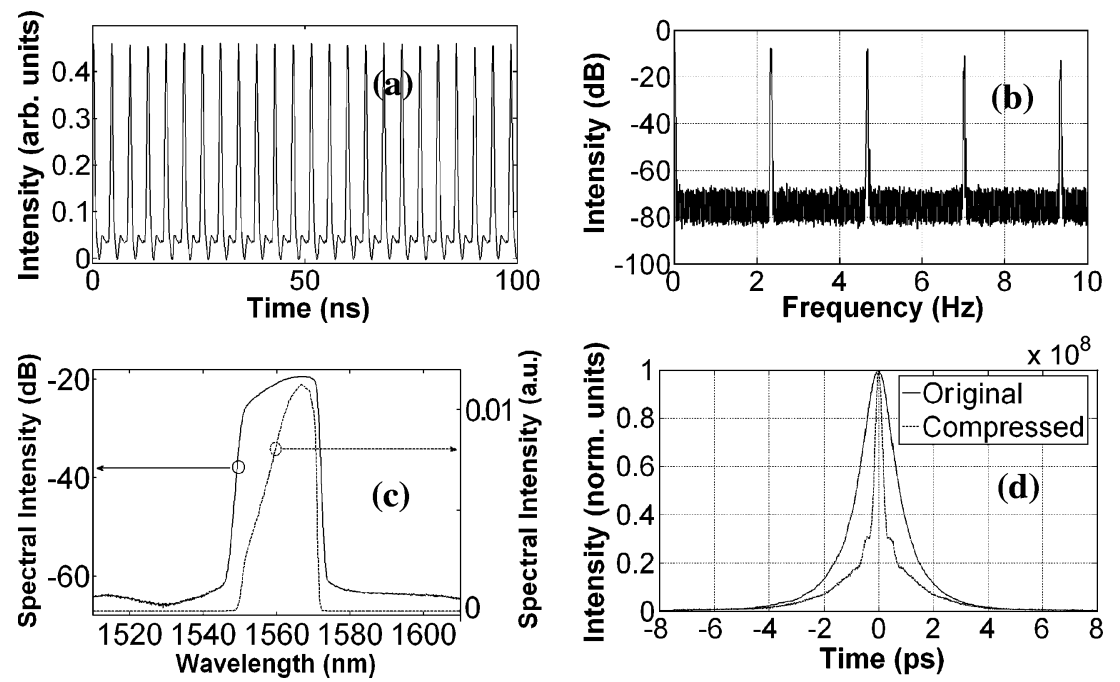

Fig. 2. Measurement of the 234-MHz BIEDF fiber laser. (a) Oscilloscope trace; (b) RF spectrum; (c) optical spectrum; (d) autocorrelation trace.

demonstrated that the pump power requirement $(450 \mathrm{~mW})$ for the gain-guided soliton mode locking is far smaller than that of conventional soliton mode locking (700 mW) [12] while both of them are suitable for the high fundamental repetition rate operation. Therefore, the cavity design presented here could also be applied to other high doping Yb-fiber/Er-Yb-co-doped-fiber/ double-cladding-Yb-fiber lasers.

\section{CONCLUSION}

We have demonstrated, for the first time, a novel high fundamental repetition rate fiber laser operating at strong normal dispersion regime. A fundamentally mode-locked gain-guided soliton fiber laser generating 1.11-ps pulses at $234 \mathrm{MHz}$ is achieved. The fiber laser design used here overcomes the obstacles for increasing the fundamental repetition rate of the fiber lasers. Successful mode locking in the normal dispersion regime makes the fiber laser cavity ubiquitous and suggests that it can be applied to other high-doping fiber lasers for high fundamental repetition rate operation.

\section{REFERENCES}

[1] J. Rauschenberger, T. M. Fortier, D. J. Jones, J. Ye, and S. T. Cundiff, "Control of the frequency comb of a mode-locked erbium doped-fiber laser," Opt. Lett., vol. 24, pp. 1404-1410, 2002.

[2] J. B. Schlager, P. D. Hale, and D. L. Franzen, "High-sensitivity optical sampling using an erbium-doped fiber laser strobe," Microw. Opt. Technol. Lett., vol. 6, pp. 835-837, 2007.

[3] W. C. Swann and N. R. Newbury, "Frequency-resolved coherent lidar using a femtosecond fiber laser," Opt. Lett., vol. 31, pp. 826-828, 2006.
[4] K. S. Abedin, N. Onodera, and M. Hyodo, "Repetition-rate multiplication in actively mode-locked fiber lasers by higher-order FM mode locking using a high-finesse Fabry-Perot filter," Appl. Phys. Lett., vol. 73, pp. 1311-1313, 1998.

[5] S. Zhou, D. G. Ouzounov, and F. W. Wise, "Passive harmonic mode locking of a soliton $\mathrm{Yb}$ fiber laser at repetition rates to $1.5 \mathrm{GHz}$," Opt. Lett., vol. 31, pp. 1041-1043, 2006.

[6] S. C. Zeller, T. Südmeyer, K. J. Weingarten, and U. Keller, "Passively modelocked $77 \mathrm{GHz}$ Er:Yb:glass laser," Electron. Lett., vol. 43, pp. 32-33, 2007.

[7] M. L. Dennis and I. N. Duling, III, "High repetition rate figure eight laser with extracavity feedback," Electron. Lett., vol. 28, pp. 1894-1896, 1992.

[8] M. E. Fermann, M. J. Andrejco, Y. Silverberg, and M. L. Stock, "Passive modelocking by using nonlinear polarization evolution in a polarizing-maining erbium-doped fiber," Opt. Lett., vol. 18, pp. 894-896, 1993.

[9] J. J. McFerran, L. Nenadovic, W. C. Swann, J. B. Schlager, and N. R. Newbury, "A passively mode-locked fiber laser at $1.54 \mu \mathrm{m}$ with a fundamental repetition frequency reaching $2 \mathrm{GHz}$," Opt. Express, vol. 15, pp. 13155-13166, 2007.

[10] F. Ilday, J. Chen, and F. Kärtner, "Generation of sub-100-fs pulses at up to $200 \mathrm{MHz}$ repetition rate from a passively mode-locked $\mathrm{Yb}$-doped fiber laser," Opt. Express, vol. 13, pp. 2716-2721, 2005.

[11] T. Wilken, T. W. Hänsch, R. Holzwarth, P. Adel, and M. Mei, "Low phase noise $250 \mathrm{MHz}$ repetition rate fiber fs laser for frequency comb applications," in Conf. Lasers and Electro-Optics/Quantum Electronics and Laser Science Conf. and Photonic Applications Systems Technologies, OSA Tech. Dig. Series (CD) (Optical Society of America, 2007), Paper CMR3.

[12] J. Chen, J. W. Sickler, E. P. Ippen, and F. X. Kärtner, "High repetition rate, low jitter, low intensity noise, fundamentally mode-locked $167 \mathrm{fs}$ soliton Er-fiber laser," Opt. Lett., vol. 32, pp. 1566-1568, 2007.

[13] L. M. Zhao, D. Y. Tang, X. Wu, H. Zhang, C. Lu, and H. Y. Tam, "Dynamics of gain-guided solitons in a dispersion-managed fiber laser with large normal cavity dispersion," Opt. Commun., vol. 281, pp. 3324-3326, 2008.

[14] A. Chong, J. Buckley, W. Renninger, and F. Wise, "All-normal-dispersion femtosecond fiber laser," Opt. Express, vol. 14, pp. 10095-10100, 2006. 